\title{
Accurate Jovian Radio Flux Density Measurements Show Ammonia to be Subsaturated in the Upper Troposphere
}

\author{
James Gibson, Wm. J. Welch, and Imke de Pater \\ Radio Astronomy Laboratory \\ University of California, Berkeley
}

May 3, 2004

\begin{abstract}
The availability of new accurate radio flux densities of Jupiter in and around the $\lambda 1.3 \mathrm{~cm}$ ammonia absorption band, one from groundbased radio data and five from the WMAP satellite, permits reexamination of the structure of the Jovian upper troposphere. These flux densities, with accuracies of $1-3 \%$, indicate that the Jovian atmospheric ammonia is globally subsaturated within and above the ammonia cloud tops, 0.4 bar $\leq \mathrm{P} \leq 0.6$ bar, and subsolar (by a factor of 2) below the cloud base, 0.6 bar $\leq \mathrm{P} \leq 2$ bar.
\end{abstract}

\section{Introduction}

\subsection{The Radio Brightness of Jupiter}

Ammonia, with its strong inversion/rotation absorption band centered at $1.3 \mathrm{~cm}$ wavelength, is the main source of opacity for the thermal spectrum from the planet Jupiter at short $\mathrm{cm}$ and $\mathrm{mm}$ wavelengths. In addition, $\mathrm{H}_{2} \mathrm{~S}$ may play a role in the emission away from the ammonia band center at $\mathrm{mm}$ wavelengths, $\mathrm{H}_{2} \mathrm{O}$ at longer $\mathrm{cm}$ wavelengths, and cloud particles $\left(\mathrm{NH}_{3}-\right.$ ice, $\mathrm{NH}_{4} \mathrm{SH}$, and $\mathrm{H}_{2} \mathrm{O}$ ) at both $\mathrm{mm}$ and $\mathrm{cm}$ wavelengths. At the longer $\mathrm{cm}$ 
wavelengths, there is an additional component of emission that comes from the planet's extended radiation belts (cf. de Pater, 1990).

Wildt (1937) first reported the detection of Jovian ammonia observed as absorption in the spectrum of sunlight reflected from the visible clouds. The first relevant radio observations of Jupiter were those of Alsop and Giordmaine (1961) at $3 \mathrm{~cm}$, Thornton and Welch (1963) at $8.35 \mathrm{~mm}$, and Kalaghan and Wulfsberg (1968) at $8.6 \mathrm{~mm}$. All of these were single antenna measurements in which the planet was not spatially resolved. Thornton and Welch were able to explain their observation with a model in which ammonia is saturated in an adiabatic atmosphere within and above the cloud tops. The ammonia absorption formulas used for their model were based on laboratory data for pure ammonia at room temperature taken from Townes and Shawlow (1955). These had to be extrapolated to lower temperatures and higher pressures in mixtures of molecular hydrogen and helium as appropriate to the Jovian atmosphere. This process was uncertain, but the antenna gain calibration error of $10 \%$ generated the dominant uncertainty in the model comparison. The conclusion that the ammonia was close to saturation near the cloud tops seemed firm, but no better modeling could be done at that time without more accurate absorption formulas and precise antenna gain calibration.

Since the 1960's, much more has been learned about Jupiter's atmosphere from ground based radio observations, as well as observations in other spectral ranges and by spacecraft. There have been further radio spectral observations as well as high angular resolution studies with instruments like the Very Large Array of the NRAO and the BIMA and OVRO millimeter wavelength arrays. In the Jovian atmosphere at altitudes above $\mathrm{P} \lesssim 0.15$ bar the ammonia abundance is expected to fall below the saturation values as a result of photochemical destruction due to the solar ultraviolet radiation (Wildt, 1937; Strobel, 1973). An accurate study of this effect by Edgington et al. (1998) based on Hubble Space Telescope observations in the ultraviolet found large departures from saturation at atmospheric pressures below 0.15 bar. The microwave measurements discussed in the present study are sensitive to the ammonia distribution at pressures between 0.4 bar and 2 bar. 


\subsection{Laboratory Data}

To support the observations, there has been a continuing effort to obtain further laboratory measurements of ammonia absorption both in the pure phase and in mixtures of $\mathrm{He}$ and $\mathrm{H}_{2}$ such as are expected for the Jovian atmosphere and to better fit the available laboratory data with theoretical line profiles. Wrixon et al. (1971) experimented with fitting both the Van Vleck-Weisskopf (Townes and Shawlow, 1955) and the Ben-Reuven (1965) line shapes to the absorption data of Bleaney and Loubser (1950). They found that the former shape fit well at low pressures near the band center and the latter fit better at high pressures, where the effective center frequency of the band shifts toward zero frequency.

Berge and Gulkis (1976) have fit the measurements of Bleaney and Loubser (1950) and Poynter and Kakar (1975) to the Ben-Reuven line shape spectrum. Their calculations seem to be accurate for pure ammonia but not so accurate for mixtures of ammonia with $\mathrm{He}$ and $\mathrm{H}_{2}$ (Spilker, 1990). At the longer wavelengths $(1.5 \mathrm{~cm}-3 \mathrm{~cm})$ the most accurate measurements of ammonia absorption in mixtures of $\mathrm{He}$ and $\mathrm{H}_{2}$ are those of Spilker $(1990,1993)$ who fits his data with a Ben-Reuven line shape. The resulting formalism appears to predict the absorption accurately to within a few percent over this range of wavelengths, but it cannot be extrapolated very far outside of this range.

For the shorter wavelengths $(0.3 \mathrm{~cm}-1 \mathrm{~cm})$ the important measurements are those of Joiner et al. (1989) and Mohammed and Steffes (2003) for 0.7 - 1.0 $\mathrm{cm}$, and Mohammed and Steffes (2004) for $0.33 \mathrm{~cm}$. These data were taken with gas mixtures corresponding to the approximate Jovian abundances, at pressures of 0.5, 1.0, and $2.0 \mathrm{bar}$, and over a range of temperatures from 180 $\mathrm{K}$ to $300 \mathrm{~K}$. The data were fit with the Ben-Reuven line shape spectrum. Comparison between these data and the formalism shows errors of the order of $20 \%$ for the wavelengths near $1 \mathrm{~cm}$ and $30 \%$ at $0.3 \mathrm{~cm}$. The errors in the absorption formulas will translate directly into errors in the abundances deduced from model fitting.

\subsection{Further Microwave Spectral Measurements of Jupiter}

Wrixon et al. (1971) reported brightness measurements at eight wavelengths over the range $0.8 \mathrm{~cm}$ to $1.5 \mathrm{~cm}$. With some effort, absolute gain calibrations 
with an accuracy of $4-5 \%$ were obtained for all of these measurements. One could see in the data the dip corresponding to the ammonia band. However, changing the model, for example, by lowering the humidity from $100 \%$ to $10 \%$, barely changes the model brightness relative to the uncertainty of the data, and it is clear that substantially better absolute antenna gain calibration is required to understand the atmospheric structure from such observations. The reason for this circumstance is that the saturation vapor pressure of ammonia is a very steep function of temperature. This follows from the vapor-pressure temperature relationship for ammonia:

$$
\mathrm{P}_{\mathrm{NH}_{3}}=1.3 \times 10^{7} \mathrm{e}^{-3753.6 / \mathrm{T}} \text { bar. }
$$

(Lasker, 1963; Thornton and Welch, 1963). $\mathrm{P}_{\mathrm{NH}_{3}}$ changes very rapidly with temperature near the cloud top temperature of about $140 \mathrm{~K}$.

One useful general result of the Wrixon et al. (1971) project was the construction of a better absolute flux density scale for other radio astronomy observations. Janssen and Welch (1973) used their equipment and technique and remeasured the flux density of Jupiter at $1.35 \mathrm{~cm}(22.285 \mathrm{GHz})$. Janssen et al. (1974) [henceforth JGW] used this measurement to determine the flux densities of some standard radio sources, such as CasA, with the same telescope, giving them an absolute flux density accurate to $4 \%$. Combining these measurements with earlier longer wavelength calibrations provided an overall scale that could be used for other observations in the short centimeter range.

\section{More Recent Radio Results at Short Wave- lengths}

Since that time there have been several investigations of the spectrum of Jupiter in the short-cm $/ \mathrm{mm}$ range. We report here on results of Klein and Gulkis (1978) [hereafter KG], and new data from the recently-launched Wilkinson Microwave Anisotropy Probe satellite (WMAP) (Page et al. 2003) and our own ground-based experiment. Several adjustments are applied to the KG and WMAP data in order to put all the measurements on a common scale and to address small inaccuracies. Since our atmospheric model is expressed in terms of brightness temperature defined by the Planck func- 
tion, we report brightness temperatures as defined in this way. Temperature defined through the Rayleigh-Jeans function has the advantage of being additive, since the Rayleigh-Jeans function is linear in temperature. Therefore, we report the adjustments and corrections as temperature increments (eg. $\mathrm{T}_{\mathrm{CMB}}, \mathrm{T}_{\text {synch }}$ ) defined through the RJ function and add them. Conversion to Planck brightness temperature is done as the final step.

\subsection{Klein and Gulkis}

Klein and Gulkis made an important multi-channel study of Jupiter using a receiver with filters at 13 wavelengths in the interval 1.5 to $1.25 \mathrm{~cm}(20$ to $24 \mathrm{GHz}$ ). Their data show smooth internal consistency (relative accuracy $\sim 1.5 \%$ ) and are tied to the absolute calibration of JGW. The shape of the ammonia absorption band center is shown clearly by these data in Figure 1, which also contains some other data (see eg. de Pater et al. 2001). In the lower panels, we show the KG data (blue), along with data from WMAP (cyan) and the datum from the present study (red). The KG data are from their Table II with the following corrections:

- In the original calibrations of Janssen and Welch (1973) no correction for the CMB radiation was made. This $2.74 \mathrm{~K}$ blackbody brightness is blocked by the planet and must be added to the flux density measured for the planet relative to the background (which does contain this extra flux). The calibration of JGW is based on this earlier work, and is therefore missing this correction. At Janssen and Welch's wavelength of $1.35 \mathrm{~cm}$, the correction to their brightness temperature of $136 \mathrm{~K}$ is $2.24 \mathrm{~K}$, an increase by a factor of 1.016. This factor must be applied to the JGW flux density scales and therefore to KG's measurements of Jupiter's flux density.

- All KG disk brightness temperatures were based upon total flux density measurements. As the authors point out, this includes Jupiter's synchrotron radiation at approximately $1 \%$ of the observed intensity. We adopt a value of 1.5 Jy for Jupiter's synchrotron radiation at $1.05 \mathrm{~cm}$ $(28.5 \mathrm{GHz})$ (see below), based upon the work by de Pater and Dunn (2003). To make the KG data consistent with ours, we re-calculate the synchrotron component at $1.5-1.25 \mathrm{~cm}$ (20-24 GHz), adopting the same slope as used by $\mathrm{KG}\left(\mathrm{S} \sim \nu^{-0.4}\right.$; $\mathrm{S}$ the flux density and $\nu$ the 
frequency) over the frequency range $20-30 \mathrm{GHz}(1.5-1.25 \mathrm{~cm})$. We further correct these numbers for the fact that the telescope used by KG overresolved Jupiter's synchrotron radiation by $4 \%$ (de Pater et al. 2003). Overall this amounts to a synchrotron radiation component of $\sim 1.6 \mathrm{Jy}, 0.2 \mathrm{Jy}$ lower than adopted by KG.

- KG (independently of Janssen and Welch) also did not correct their flux densities for the blockage of the CMB by Jupiter. We compute and add the correction terms appropriate for each frequency.

- We convert to Planck brightness temperature, as noted above.

The original KG disk temperatures, these corrections, and the derived values of brightness temperature are listed in Table I.

Finally, it should be noted that all of the flux densities in KG's Table II are uncertain by the absolute $4 \%$ uncertainty of the JGW flux density calibration, and this uncertainty is added in quadrature to the KG uncertainties listed.

\subsection{The WMAP Jovian Flux Densities}

Page et al. (2003) describe the radio brightness measurements of Jupiter that were obtained in the course of on-orbit calibrations during the first year of background measurements made with the WMAP satellite. The windows cover the wavelength range from about $3 \mathrm{~mm}$ to $13 \mathrm{~mm}$. The uncertainties are in the range $1-3 \%$.

- The WMAP bands are wide, so that the effective center frequency depends on the spectrum of the observed object. Page et al. report effective frequencies and bandwidths appropriate to a variety of source spectral distributions. We start with their values for a thermal spectrum and from those compute new effective frequencies based on the deviations from thermal predicted by our model. We find small changes in effective frequency, negligible change in effective bandwidth.

- As before, the blocking of the CMB by Jupiter is corrected by adding the (Rayleigh-Jeans) brightness temperature of the CMB at each frequency. Page et al. give these values in their section 2.4 and we list them in Table II. 
- For the synchrotron component between 1.5 and $1.0 \mathrm{~cm}$ (20 and 30 $\mathrm{GHz}$ ), we adopt the KG law of $\mathrm{S} \sim \nu^{-0.4}$. The numbers become increasingly unreliable with increasing frequency, and we have adopted $0.5 \mathrm{Jy}$ at $0.73 \mathrm{~cm}(41 \mathrm{GHz})$, and zero at $0.40 \mathrm{~cm}(61 \mathrm{GHz})$ and 0.32 cm (93 GHz).

- Finally, the corrections are added and the result is converted to Planck brightness temperature. Here, because of the large bandwidth, the value reported is that temperature which yields the appropriate flux when the Planck function is integrated over the bandwidth.

\subsection{New Ground Based Result}

Gibson (2003) has calibrated the gain of one of the BIMA millimeter array antennas (Welch et al. 1996) with an absolute accuracy of $1.4 \%$ which enabled the measurement of the Jovian flux density at $1.05 \mathrm{~cm}(28.5 \mathrm{GHz})$ with an accuracy of $1.6 \%$. The calibration technique used the signal from Jupiter to transfer the gain of a standard horn antenna to one of the antennas of the interferometer array. The comparison used all of the 10 antennas of the array for better signal to noise. The interferometry offered a number of advantages.

- The astronomical correlator served as the relative power detector.

- The $800 \mathrm{MHz}$ receiver bandwidth ensured a correlation path length of only about $30 \mathrm{~cm}$, so that the usual problems of multipath propagation and scattering to the horn were eliminated.

- The array is designed to work well at $1 \mathrm{~mm}(300 \mathrm{GHz})$, so that at $1.05 \mathrm{~cm}(28.5 \mathrm{GHz})$, the antennas are nearly perfect. The $\leq 30 \mu \mathrm{m}$ surface has no Ruze (1966) loss, elevation gain changes are negligible, and pointing errors are negligible.

A subsequent single dish measurement of the flux density of Jupiter using the calibrated antenna and standard waveguide black body reference loads produced the Jovian disk temperature of $142.9 \pm 2.3 \mathrm{~K}(1.65 \%)$ for $1.05 \mathrm{~cm}$ $(28.5 \mathrm{GHz})$. This brightness has been corrected for the blocked $2.74 \mathrm{~K}$ cosmic background radiation and a $1 \%$ contribution from the synchrotron radiation (de Pater and Dunn, 2003). The accuracy of this brightness measurement is 
nearly three times better than previous ground based measurements in this wavelength range.

\section{Model Calculations}

The model calculations here are based on the work of de Pater et al. (2001; hereafter dPGal), though we use improved formalisms for the absorption by $\mathrm{H}_{2} \mathrm{O}$ and $\mathrm{H}_{2} \mathrm{~S}$ (from DeBoer and Steffes, 1996). Also, as discussed above, we use Spilker's formalism for ammonia absorption at wavelengths longer than $1.5 \mathrm{~cm}$ and that of Joiner and Steffes (1991) and Mohammed and Steffes (2004) for wavelengths shorter than $1.5 \mathrm{~cm}$. The goal is to find the best model fit to Jupiter's radio spectrum for wavelengths between $1.5 \mathrm{~cm}$ and $0.32 \mathrm{~cm}$, the range covered by $\mathrm{KG}$, the WMAP values, and our measurement. Jupiter is not spatially resolved in any of the observations discussed here, so that we get a fit which corresponds to the global averaged atmosphere. Over this wavelength range, the ammonia absorption is sufficiently strong that we are sensitive to atmospheric structure only at altitudes above which the pressure is about 2 bar. This pressure range is included in the laboratory measurements discussed above. For the lower atmosphere we use dPGal's 'best fit radio model', a thermochemical equilibrium model, where, below all cloud layers, $\mathrm{NH}_{3}$ is enhanced by a factor of 1.2 relative to solar $\mathrm{N}$, and $\mathrm{H}_{2} \mathrm{O}$ and $\mathrm{H}_{2} \mathrm{~S}$ by factors of $4-5$ over solar $\mathrm{O}$ and $\mathrm{S}$. These enhancements are relative to the solar elemental ratios as given by Anders and Grevesse (1989) and Grevesse et al. (1991). ${ }^{1}$ The Galileo probe measured enhancements in the $\mathrm{NH}_{3}$ abundance by a factor of $3-3.5$ at $\mathrm{P}>8$ bar. (Folkner et al. 1998; Wong et al. 2003). As shown by de Pater et al. (2004), this enhancement has negligible effect at wavelengths between 1.5 and $0.32 \mathrm{~cm}$, and is therefore ignored in our study.

While there are no laboratory measurements of the absorption of solid ammonia at $\mathrm{cm}$ wavelengths, we include the effect of absorption by Jovian ammonia ice by assuming the imaginary part of the ammonia ice refractive index to be a frequency independent .01, similar to that of water ice. For par-

\footnotetext{
${ }^{1}$ We use the solar elemental ratios for N, O, and S from Anders and Grevesse (1989) and for $\mathrm{C}$ from Grevesse et al. (1991). The solar $\mathrm{N} / \mathrm{H}$ value is $1.12 \times 10^{-4}$. The volume mixing ratio or mole fraction of solar nitrogen in Jupiter's atmosphere is $1.97 \times 10^{-4}$. We use the word abundance to mean volume mixing ratio or mole fraction.
} 
ticles small compared to wavelength this gives a mass absorption coefficient that is inversely proportional to wavelength. We also assume the maximum mass of cloud ice consistent with the overall ammonia abundance (see de Pater and Mitchell, 1993 for details).

For the upper troposphere $(\mathrm{P}<2$ bar), we vary the ammonia density distribution to provide the best fit to our spectral observations using the temperature-pressure distribution of dPGal. ${ }^{2}$ The latter is shown in Figure 2b. The expected ammonia distribution divides naturally into two layers. For $\mathrm{P}<0.6$ bar, it will be maximally bounded by the saturation curve given in the equation above. For $\mathrm{P}>0.6$ bar it is expected to be more uniformly mixed. This break in distributions is evident in the solid curve in Figure 2a. The clouds and distributions of the $\mathrm{H}_{2} \mathrm{O}$ and $\mathrm{H}_{2} \mathrm{~S}$ gases of the model lower atmosphere are also shown. In addition to the temperature-pressure curve, Figure $2 \mathrm{~b}$ shows the spectral weighting functions at several wavelengths. These show that the ammonia distribution in the saturated layer dominates the spectrum near the $1.23 \mathrm{~cm}$ wavelength spectral minimum. The $7 \mathrm{~mm}$ and $3.3 \mathrm{~mm}$ weighting functions indicate the sensitivity to ammonia in the 0.6 to 2 bar layer of the atmosphere.

In Figure 1 the results of a variety of models appear superposed over the data sets. The top panel shows the model parameters and the model curves and all the available data. The contributions of the two ammonia layers are treated differently in these models. For the lower layer $(0.6<\mathrm{P}<2$ bar $)$, the uniformly mixed fractions are varied. For the upper layer the mixing fraction is held constant while the humidity is varied. In the top panel the errors of the earlier data are so large that all the models appear to fit equally well. In the central panel we have enlarged the $0.3-3 \mathrm{~cm}$ region, and between 0.3 $\mathrm{cm}$ and $1.5 \mathrm{~cm}$ we show only the KG data (blue), the WMAP values (cyan), and our own measurement (red). The bottom two panels separate the region of the central minimum from the broader region of the shorter wavelengths. This division, as noted, permits us to consider the fit of the spectral data to the two ammonia layers separately.

\footnotetext{
${ }^{2}$ The brightness temperature is not very sensitive to the temperature-pressure profile within and above the ammonia-ice clouds, since the vapor pressure is coupled to the physical temperature of the atmosphere. For example, an increase in this physical temperature will result in greater optical depth of $\mathrm{NH}_{3}$, and thus emission from a higher (and cooler) region.
} 
In the bottom left panel, the light blue and green lines represent the extreme fractional ammonia abundances of $7 \times 10^{-5}$ and $2 \times 10^{-4}$ respectively. We see that the WMAP data excludes these values, favoring the red, dark blue, or dashed lines corresponding to $1 \times 10^{-4}$, the best fit with an error estimate of $\pm 0.25 \times 10^{-4}$. This estimate includes the error in ammonia absorption coefficient discussed above. It also includes the uncertainty in the effect of the clouds. The ice cloud opacity is inversely proportional to wavelength and has a maximum effect at the $0.3 \mathrm{~cm}$ wavelength end of the spectrum. ${ }^{3}$ The uncertainty of the cloud effect is small compared with the uncertainties of the ammonia gas opacity. This conclusion about the abundance in the region $0.6<\mathrm{P}<2$ bar also agrees with the findings of de Pater (1986) and dPGal, which were based on data in the $2-6 \mathrm{~cm}$ wavelength range using an independent technique (limb-darkening profile fitting).

The bottom right panel shows the region of the spectral minimum. The dark blue line is a calculation for dPGal's best fit radio model with the fractional abundance set to $1 \times 10^{-4}$, assuming Jovian air to be completely saturated within and above the ammonia-ice cloud layer (i.e., humidity is $100 \%$ ). For this model, the ammonia abundance follows the saturated vapor curve at pressure $\mathrm{P}<0.6$ bar, where the temperature is low enough for $\mathrm{NH}_{3}$ to condense out. This is the maximum concentration of $\mathrm{NH}_{3}$ gas that could be present in the atmosphere within and above the $\mathrm{NH}_{3}$-ice cloud. The red lines present similar calculations, but for different humidities (at $\mathrm{P}<0.6$ bar): the solid curve is for a $10 \%$ humidity, the top dashed curve for $1 \%$ and the bottom dashed curve for $50 \%$. It is clear that the atmosphere must be dry for any model to match our very accurate $1.05 \mathrm{~cm}$ point and the $\mathrm{KG}$ data points. For the green and cyan lines we adopted a $10 \%$ humidity, and different ammonia abundance at $\mathrm{P}<2$ bar: for the green line we adopted a $\mathrm{NH}_{3}$ abundance of $1 \times 10^{-4}$ (dotted line in Fig. 1), and for the cyan of $7.0 \times 10^{-5}$ (dot-dash line in Fig. 1).

We have shown above that the $\mathrm{NH}_{3}$ abundance, on average, is subsaturated by at least a factor of 2 at $\mathrm{P}<0.6$ bar. Such a global average can, for example, be brought about by a difference in humidity between zones and belts, as first suggested by KG. Because the zones are usually characterized by a net upwelling, the abundance profiles above zones likely follow the sat-

\footnotetext{
${ }^{3}$ The modeled opacity of the clouds is plausible, but even if it were left out, the green and light blue curves would be raised by only $2-3 \mathrm{~K}$ at $0.3 \mathrm{~cm}$ wavelength.
} 
urated vapor pressure curves within and above the cloud layers (i.e., 100\% $\mathrm{RH})$. Belts are characterized by subsidence, and hence are likely subsaturated in $\mathrm{NH}_{3}$ gas. Our results show the average of both types of regions, and thus support this general circulation model.

\section{Conclusions and Future Work}

A comparison of the model calculations with the radio data between $3 \mathrm{~mm}$ up to $1.5 \mathrm{~cm}$ clearly show that:

- The atmosphere is subsaturated ( $\lesssim 50 \%$ humidity) on a global average in the pressure range $0.4<\mathrm{P}<0.6$ bar.

- At pressures $0.6<\mathrm{P}<2$ bar, the ammonia abundance is subsolar at $\sim 1.0 \times 10^{-4} \pm .25 \times 10^{-4}$. (i.e., a factor of $\sim 2$ below solar $\mathrm{N}$ ).

With improved accuracy in the Jupiter data, an important source of uncertainty becomes the synchrotron radiation. In the past this contribution has either been ignored, or simply taken as $\sim 1 \%$ of the total emission - a reasonable estimate. However, with error bars now down to the $1 \%$ level, accurate measurements of Jupiter's synchrotron radiation are needed. Only two such measurements exist at present, which differ by a factor of 3.5. Bolton et al. (2002) observed Jupiter's synchrotron radiation with the Cassini spacecraft at a frequency of $13.8 \mathrm{GHz}$, and report a total flux density of $0.44 \pm 0.15$ Jy. De Pater and Dunn (2003) observed the synchrotron radiation with the VLA at frequencies of 14.95 and $22.46 \mathrm{GHz}$, and found total flux densities of $1.5 \pm 0.15 \mathrm{Jy}$ and $1.5 \pm 0.5 \mathrm{Jy}$, respectively. The latter authors were able to fit the VLA data with model calculations, but not the Cassini data point. They conclude their paper by saying that the 'discrepancy between the VLA and Cassini data is puzzling and intriguing', and recommend frequent observations at these high frequencies to establish whether or not the radiation varies over time or is stable at these high frequencies. From the perspective of Jupiter's atmosphere, we support their conclusion, and intend to take the first steps towards re-observing Jupiter at $10-40 \mathrm{GHz}$ with the VLA. A second source of uncertainty is in the accuracies of the ammonia absorption coefficients and the absorption by the ammonia ice. These uncertainties are included in the overall accuracy estimates of the model fitting, and the conclusions that the ammonia is both below saturation in the highest layer by a 
factor of at least two and below solar abundance down to the level of 2 bar by a factor of two seem secure. However, when (better) laboratory data are available for both components, a more accurate model can be made from the astronomical data.

\section{Acknowledgements}

IdP thanks Paul Romani and David DeBoer for help with the model calculations, and the Miller Institute for Basic Research in Science at the University of California in Berkeley for support during this research. This work was further funded in part by NASA grant NAG5-12062 to the University of California, Berkeley. WJW acknowledges support from NASA grant NAG5-10657 and NSF grant AST-0228963.

\section{Bibliography}

- Alsop, L. and J. A. Giordmaine 1961. Columbia Radiation Lab Report, DA-36-039-SC-78330.

- Anders, E. and N. Grevesse 1989. Abundances of the Elements - Meteoric and Solar Geochim. et Cosmochim. Acta 53, 197.

- Ben-Reuven, A. 1965. Transition from Resonant to Nonresonant Line Shape in Microwave Absorption. Phys. Rev. Letters. 14, 349.

- Berge, G. L. and S. Gulkis 1976. Earth-Based Radio Observations of Jupiter: Millimeter to Meter Wavelengths. Jupiter T. Gehrels, ed. University of Arizona Press. 621.

- Bleaney, B. and J. H. N. Loubser 1950. The Inversion Spectra of $\mathrm{NH}_{3}$, $\mathrm{CH}_{3} \mathrm{Cl}$ and $\mathrm{CH}_{3} \mathrm{Br}$ at High Pressures. Proc. Phys. Soc. 77, 418.

- Bolton, S. J. and 20 co-authors, 2002. Ultra-relativistic Electrons in Jupiter's Radiation Belts. Nature 415, 987.

- DeBoer, D. R. and P. G. Steffes 1996. Estimates of the Tropospheric Vertical Structure of Neptune Based on Microwave Radiative Transfer Studies. Icarus 123, 342 
- de Pater, I. and 14 co-authors, 2003. Jupiter's Radio Spectrum from $74 \mathrm{MHz}$ up to $8 \mathrm{GHz}$. Icarus 163, 434.

- de Pater, I., 1986, Jupiter's Zone-Belt Structure at Radio Wavelengths: II. Comparison of Observations with Model Atmosphere Calculations, Icarus, 68, 344-365.

- de Pater, I. 1990. Radio Images of the Planets. Ann. Rev. A\&A. 28, 347.

- de Pater, I. and D. L. Mitchell 1993. Radio Observations of the Planets - The Importance of Laboratory Measurements. Journ. Geophys. Res. 98, 5471 .

- de Pater, I., D. Dunn, P. Romani and K. Zahnle 2001. Reconciling Galileo Probe Data and Ground-Based Radio Observations of Ammonia on Jupiter. Icarus 149, 66.

- de Pater, I. and D. Dunn 2003. VLA Observations of Jupiter's Synchrotron Radiation at 15 and 22 GHz. Icarus 163, 449.

- de Pater, I., D. R. DeBoer, M. Marley, R. Freedman, and R. Young, 2004. Retrieval of Water in Jupiter's Deep Atmosphere using Microwave Spectra of its Brightness Temperature. Icarus, submitted.

- Edgington, S. G. and 7 co-authors 1998. On the Latitude Variation of Ammonia, Acetylene, and Phosphine Altitude Profiles on Jupiter from HST Faint Object Observations. Icarus 133, 192.

- Folkner, W. M. R. Woo and S. Nandi 1998. Ammonia Abundance in Jupiter's Atmosphere Derived from the Attenuation of the Galileo Probe's Radio Signal. Journ. Geophys. Res. Planets 103, 22847.

- Gibson, J. L. 2003. An Accurate Measurement of the Brightness Temperature of Jupiter at $28.5 \mathrm{GHz}$. Ph.D. Dissertation, University of California, Berkeley.

- Grevesse, N. and 5 co-authors 1991. Vibration-Rotation Bands of CH in the Solar Infrared Spectrum and the Solar Carbon Abundance. Astron. \& Astrophys. 242, 488. 
- Janssen, M. A., and W. J. Welch 1973. Mars and Jupiter: Radio Emission at $1.35 \mathrm{~cm}$. Icarus 18, 502 .

- Janssen, M. A. J., L. Golden and W. J. Welch 1974. Extension of the Absolute Flux Density Scale to $22.285 \mathrm{GHz}$. Astron. \& Astrophys. 33, 373.

- Joiner, J. and P. G. Steffes 1991. Modeling of Jupiter's Millimeter Wave Emission Utilizing Laboratory Measurements of Ammonia $\left(\mathrm{NH}_{3}\right)$ Opacity. Journ. Geophys. Res. 96, 17463.

- Kalaghan, P. M. and K. N. Wulfsberg, 1968. Radiometric Observations of the Planets Jupiter, Venus, and Mars at a Wavelength of $8.6 \mathrm{~mm}$. Astrophys. J. 154, 771.

- Klein, M. J. and S. Gulkis 1978. Jupiter's atmosphere - Observations and Interpretation of the Microwave Spectrum Near 1.25-cm Wavelength. Icarus 35, 44.

- Kuiper, G. P. 1952. Atmospheres of the Earth and Planets. University of Chicago Press, Chicago

- Lasker, B. M., 1963. Wet Adiabatic Model Atmospheres for Jupiter. Astrophys. Journ. 138, 709.

- Mohammed, P. N. and P. Steffes 2003. Laboratory Measurements of the Ka-band $(7.5 \mathrm{~mm}$ tp $9.2 \mathrm{~mm})$ opacity of phosphine $\left(\mathrm{PH}_{3}\right)$ and ammonia $\left(\mathrm{NH}_{3}\right)$ under simulated conditions for the Cassini-Saturn encounter. Icarus 166, 425 .

- Mohammed, P. N. and P. Steffes 2004. preprint

- Page, L. and 13 co-authors 2003. First-Year Wilkinson Microwave Anisotropy Probe (WMAP) Observations: Beam Profiles and Window Functions. Astrophys. Journ. Suppl. 148, 39.

- Poynter, R. L. and R. K. Kakar 1975. The Microwave Frequencies, Line Parameters and Spectral Constants for ${ }^{14} \mathrm{NH}_{3}$. Astrophys. J. Suppl. 29, 87 .

- Ruze, J. 1966. Antenna Tolerance Theory - A Review Proc. of the IEEE 54, 633. 
- Spilker, T. R. 1990. Laboratory Measurements of Microwave Absorptivity and Refractivity Spectra of Gas Mixtures Applicable to Giant Planet Atmospheres. Ph.D. Dissertation, Stanford University

- Spilker, T. R. 1993. New Laboratory Measurements on Ammonia's Inversion Spectrum, With Implications for Planetary Atmospheres. Journ. Geophys. Res. 98, 5539 .

- Thornton, D. D. and W. J. Welch 1963. 8.35 mm Radio Emission from Jupiter. Icarus 2, 228.

- Townes, C. and A. Shawlow, 1955. Microwave Spectroscopy. McGrawHill

- Welch, W. J. and 36 co-authors 1996. The Berkeley-Illinois-MarylandAssociation Millimeter Array. PASP 108, 93.

- Wildt, R. 1937. Photochemistry of Planetary Atmospheres. Astrophys. J. $\mathbf{8 6}, 321$.

- Wong, M. H., P. R. Mahaffy, S. K. Atreya, H. B. Niemann and T. C. Owen 2003. Updated Galileo Probe Mass Spectrometer Measurements of Carbon, Oxygen, Nitrogen, and Sulfur on Jupiter. Icarus, submitted.

- Wrixon, G. T., Welch, W. J. and D. D. Thornton 1971. The Spectrum of Jupiter at Millimeter Wavelengths. Astrophys. J. 169, 171. 

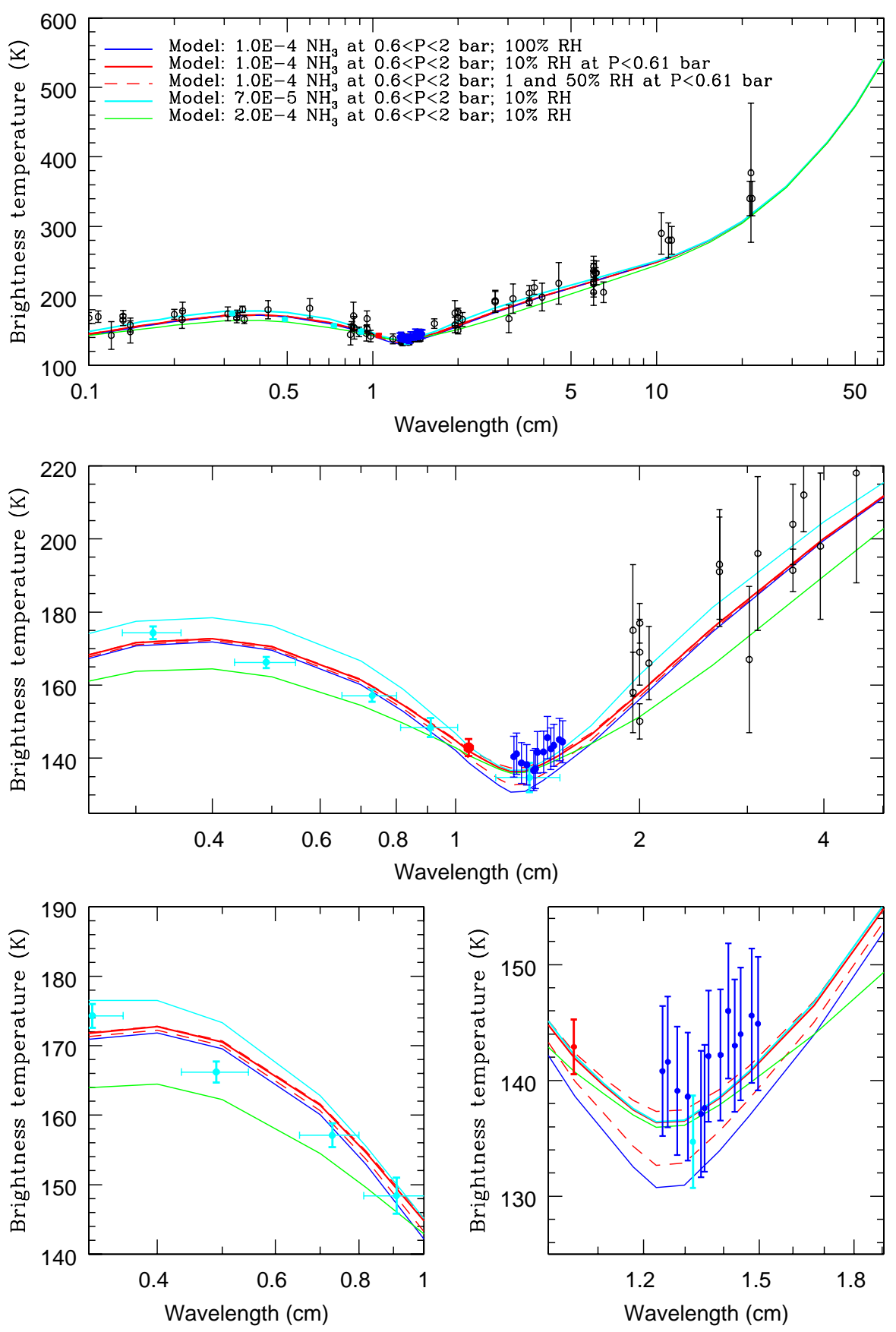

Figure 1: Jupiter microwave measurements and model spectra. For details, see text. 

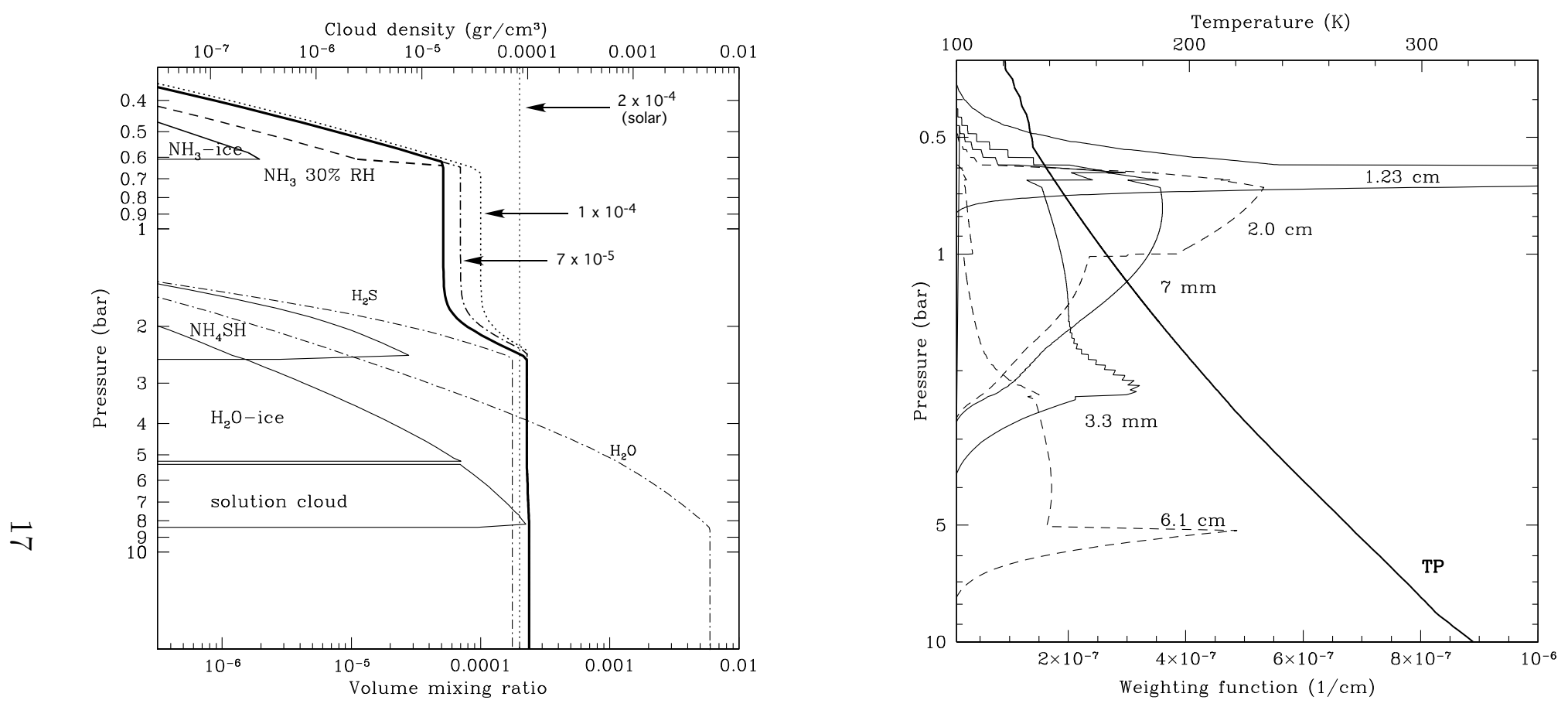

Figure 2: These figures are adapted from de Pater et al. (2001). The left shows the various cloud layers for the radio best fit model, the region above 0.6 bar with relative humidities of $100 \%$ and $30 \%$ and the layer with $0.6<\mathrm{P}<2$ bar with ammonia mixing fractions of $7 \times 10^{-5}, 1 \times 10^{-4}$, and $2 \times 10^{-4}$. The right shows the temperature-pressure curve (TP) and the spectral weighting functions. The left shows various abundance profiles for $\mathrm{NH}_{3}$ gas: the solid line is the profile derived by dPGal; the dotted line is the best fit to the WMAP values, and the dashed curve shows an abundance that is too low compared to the WMAP values. The $\mathrm{NH}_{3}$ solar abundance is indicated. The solid $\mathrm{NH}_{3}$ profile follows the saturated vapor curve at $\mathrm{P}<0.6$ bar. We also show the abundance profiles for $\mathrm{H}_{2} \mathrm{O}$ and $\mathrm{H}_{2} \mathrm{~S}$. 
Table I: Modifications to KG Data

\begin{tabular}{|c|c|c|c|c|c|c|c|c|c|}
\hline$\lambda$ & $\nu$ & $\begin{array}{l}\mathrm{T}_{\mathrm{d}}{ }^{1} \\
\text { orig. }\end{array}$ & $\begin{array}{c}\mathrm{S}_{\mathrm{t}}{ }^{2} \\
\text { corrected } \\
(* 1.016)\end{array}$ & $\begin{array}{c}\mathrm{S}_{\mathrm{nt}}{ }^{3} \\
\text { corrected } \\
(-0.2)\end{array}$ & $\begin{array}{l}\mathrm{T}_{\mathrm{d}}{ }^{4} \\
\text { new }\end{array}$ & $\begin{array}{c}\mathrm{KG} \\
\mathrm{CMB} \\
\text { correction }\end{array}$ & $\begin{array}{c}\text { RJ to } \\
\text { Planck } \\
\text { correction }\end{array}$ & $\begin{array}{c}\mathrm{T}_{\mathrm{b}} \\
\text { final }\end{array}$ & $\sigma^{5}$ \\
\hline $\mathrm{cm}$ & $\mathrm{GHz}$ & $\bar{K}$ & $\mathrm{Jy}$ & $\mathrm{Jy}$ & K & K & K & $\mathrm{K}$ & $\bar{K}$ \\
\hline 1.4953 & 20.050 & 139.6 & 73.43 & 1.67 & 142.14 & 2.29 & 0.503 & 144.9 & 5.8 \\
\hline 1.4772 & 20.295 & 140.3 & 75.52 & 1.66 & 142.80 & 2.28 & 0.51 & 145.6 & 5.8 \\
\hline 1.4459 & 20.735 & 138.8 & 77.88 & 1.64 & 141.21 & 2.27 & 0.521 & 144.0 & 5.8 \\
\hline 1.4297 & 20.970 & 137.9 & 79.07 & 1.64 & 140.21 & 2.27 & 0.526 & 143.0 & 5.7 \\
\hline 1.4125 & 21.225 & 140.7 & 82.65 & 1.63 & 143.22 & 2.26 & 0.533 & 146.0 & 5.8 \\
\hline 1.3912 & 21.550 & 136.9 & 82.89 & 1.62 & 139.35 & 2.26 & 0.541 & 142.2 & 5.7 \\
\hline 1.3593 & 22.055 & 136.9 & 86.67 & 1.60 & 139.28 & 2.24 & 0.554 & 142.1 & 5.7 \\
\hline 1.3492 & 22.220 & 132.5 & 85.19 & 1.59 & 134.84 & 2.24 & 0.558 & 137.6 & 5.5 \\
\hline 1.3402 & 22.370 & 131.9 & 85.95 & 1.59 & 134.25 & 2.24 & 0.562 & 137.1 & 5.5 \\
\hline 1.3066 & 22.945 & 133.4 & 91.36 & 1.57 & 135.81 & 2.23 & 0.576 & 138.6 & 5.5 \\
\hline 1.2806 & 23.410 & 133.9 & 95.38 & 1.56 & 136.33 & 2.22 & 0.588 & 139.1 & 5.6 \\
\hline 1.2576 & 23.840 & 136.4 & 100.60 & 1.54 & 138.76 & 2.21 & 0.598 & 141.6 & 5.7 \\
\hline 1.2440 & 24.100 & 135.7 & 102.20 & 1.54 & 137.97 & 2.2 & 0.605 & 140.8 & 5.6 \\
\hline
\end{tabular}

1. Klein \& Gulkis Table II

2. Ibid. Factor 1.016 to account for JGW CMB correction (see text)

3. Ibid. -0.2 Jy to normalize KG synchrotron component to level used by GW (see text)

4. $\mathrm{T}_{\mathrm{d} \text { new }}=\frac{\left(\mathrm{S}_{\mathrm{t}}-\mathrm{S}_{\mathrm{nt}}\right) \lambda^{2}}{2 \mathrm{k} \Omega_{\text {jup }}}$

5. $4 \%$ absolute calibration error (from JGW) dominates. 
Table II: Modifications to WMAP Data

\begin{tabular}{|c|c|c|c|c|c|c|c|c|c|c|c|}
\hline$\lambda$ & $\nu$ & $\nu_{\text {eff }}{ }^{1}$ & $\Delta \nu$ & $\begin{array}{c}\mathrm{T}_{\mathrm{d}}{ }^{2} \\
\text { orig }\end{array}$ & $\begin{array}{c}\mathrm{T}_{\mathrm{CMB}}{ }^{3} \\
\text { correction }\end{array}$ & $\mathrm{S}_{\text {synch }}{ }^{4}$ & $\mathrm{~T}_{\text {synch }}$ & $\begin{array}{c}\mathrm{T}_{\mathrm{d}}{ }^{5} \\
\text { new }\end{array}$ & $\begin{array}{c}\text { RJ to } \\
\text { Planck } \\
\text { correction }\end{array}$ & $\begin{array}{c}\mathrm{T}_{\mathrm{b}}{ }^{7} \\
\text { final }\end{array}$ & $\sigma^{8}$ \\
\hline $\mathrm{cm}$ & $\mathrm{GHz}$ & $\mathrm{GHz}$ & $\mathrm{GHz}$ & $\mathrm{K}$ & $\mathrm{K}$ & $\mathrm{Jy}$ & $\mathrm{K}$ & $\mathrm{K}$ & $\mathrm{K}$ & $\mathrm{K}$ & $\mathrm{K}$ \\
\hline & & & & & & & & & & & \\
1.315 & 22.79 & 22.44 & 5.5 & 134.4 & 2.2 & 1.4 & 2.27 & 134.3 & 0.54 & 134.7 & 4 \\
0.9096 & 32.96 & 32.63 & 7.0 & 146.6 & 2.0 & 1.0 & 0.77 & 147.8 & 0.79 & 148.4 & 2.0 \\
0.7331 & 40.89 & 40.50 & 8.3 & 154.7 & 1.9 & 0.5 & 0.25 & 156.4 & 0.98 & 157.1 & 1.7 \\
0.4888 & 61.34 & 60.57 & 14.0 & 163.8 & 1.5 & 0.0 & 0.0 & 165.3 & 1.46 & 166.2 & 1.5 \\
0.3195 & 93.82 & 92.71 & 20.5 & 171.8 & 1.1 & 0.0 & 0.0 & 172.9 & 2.23 & 174.3 & 1.7 \\
\hline
\end{tabular}

1. Mean of passband weighted by Jupiter temperature model

2. From Page et al. section 2.4

3. Ibid

4. Synchrotron radiation component (see text)

5. $\mathrm{T}_{\mathrm{d} \text { orig }}+\mathrm{T}_{\mathrm{CMB}}-\mathrm{T}_{\text {synch }}$

6. $T_{\text {Planck }}-T_{R J}$, where $T_{\text {Planck }}$ is that temperature which yields the proper flux via the Planck function, integrated over the bandwidth

7. This is $T_{\text {Planck }}$

8. Standard error of $\mathrm{T}_{\mathrm{b} \text { final. }}$ Page et al. ibid. 